\title{
Genotype-Tissue Expression Program
}

National Cancer Institute

\section{Source}

National Cancer Institute. Genotype-Tissue Expression Program. NCI Thesaurus. Code C126654.

A program sponsored by the National Institutes of Health's Common Fund, designed to provide insights into the mechanisms of gene regulation by studying human gene expression and regulation in multiple tissues from healthy individuals, exploring diseaserelated perturbations in a variety of human diseases, and examining sexual dimorphisms in gene expression and regulation in multiple tissues. Genetic variation between individuals will be examined for correlation with differences in gene expression level to identify regions of the genome that influence whether and how much a gene is expressed. 